\title{
Test and Treat in Washington, DC: Evaluating the Costs and Benefits of a Comprehensive Strategy to Fight HIV/AIDS
}

\author{
By Estelle R. Raimondo
}

This paper assesses the economic implications of an innovative approach to preventing the spread of HIV/AIDS in the Washington, DC. "Test \& Treat" is premised on the idea that the epidemic can ultimately be eliminated by testing people widely and regularly and by putting all infected persons on antiretroviral medicines upon diagnosis. The relative costs and benefits of Test \& Treat are quantified, monetized, and compared to the status quo, which can be characterized as a 'test and wait' approach. This costbenefit analysis concludes that under a plausible set of circumstances, and with a conservative estimate of the number of infections averted, the benefits of Test \& Treat in Washington, DC would outweigh the costs of implementation.

\section{Introduction}

Washington, DC remains the American city with the highest prevalence rate of HIV/AIDS. According to its Department of Health's (DOH) 2012 annual report, the epidemic affects approximately 2.7 percent of Washington, DC's population, which puts the city on par with a number of developing countries and largely above the Center for Disease Control and Prevention's (CDC) defined rate of "generalized epidemic," or 1 percent (HAHSTA 2012). While in the past decade there have been renewed efforts to address the epidemic, with some improvements in the number of diagnoses and individuals linked to care, many challenges remain. Chief among them are structural issues such as poverty and limited access to health care in the populations most at risk of infection. Drug use and rates of other sexually transmitted infections (STI) remain high in the district. Finally, stigma and limited knowledge of HIV/AIDS status are key obstacles to an effective diagnosis-treatment strategy (Kaiser Family Foundation 2012).

The World Health Organization and the U.S. National Institutes of Health (NIH) are considering an innovative strategy, which has the potential to significantly halt the spread of the epidemic. The Test and Treat (T\&T) strategy consists of screening widely for HIV/AIDS, linking individuals to care automatically upon diagnosis and ensuring adherence to powerful antiretroviral medicines, which have been proven to reduce transmission. The ex ante cost-benefit analysis presented here assesses the economic implications of T\&T implementation in Washington, DC. First, a detailed description of the T\&T intervention is laid out and compared to the status quo. Then, the relevant costs and benefits are quantified and monetized, and the outcomes are compared. Finally, a sensitivity analysis is presented before concluding with a series of policy recommendations.

\section{Background}

Although there is still no vaccine against HIV/AIDS, significant progress in the medical realm has helped curb viral 
transmission and improve the treatment of patients infected with HIV/AIDS. One of the most important breakthroughs in recent years is the finding that HIV-infected patients who are on anti-retroviral treatment (ARV) have a 92 percent risk reduction of transmitting the virus to a noninfected partner (Attia et al. 2009). These findings are consistent with the concept of "treating our way out of the epidemic," which has prevailed since the mid-1990s (Granich et al. 2009). Furthermore, there is now a wide consensus around the idea that no single magic bullet can bring the epidemic to a standstill and that any prevention and treatment strategy must be comprehensive. The underlying principle of comprehensive care is central to most HIV/AIDS local and state policies, as well as the U.S. National Strategy on HIV/ AIDS, developed by the Obama Administration in 2010 with the overarching goal of reaching a "generation free of AIDS" (The White House 2010).

$\mathrm{T} \& \mathrm{~T}$ is an innovative approach whose goals are two-fold: improving health outcomes for people living with
HIV/AIDS, especially those who are not aware of their HIV-positive status; and reducing the HIV/AIDS incidence rate in Washington, DC. The incidence rate measures the number of new infections within a given year, usually expressed as a ratio of the number of new infections per 100,000 inhabitants. The second objective is based on evidence that ARV reduces the risk of transmission and that people who are aware of their status are also less likely to engage in risky behaviors.

The premise of this approach can be summed up as follows: HIV/AIDS can be eliminated if the population, especially people at risk, is tested on a regular basis, and if all infected persons are immediately put on antiretroviral medicines upon diagnosis, no matter their CD4 count-a measure of blood cells per micro-liter used to determine treatment initiation. As laid out in Figure 1, this is one of the most salient distinctions between T\&T and the status quo, which can be characterized as a "test and wait" approach. Indeed, for several years the CDC guidelines recommended starting ARV treatment at a CD4

Figure 1: Test \& Treat and the Counterfactual
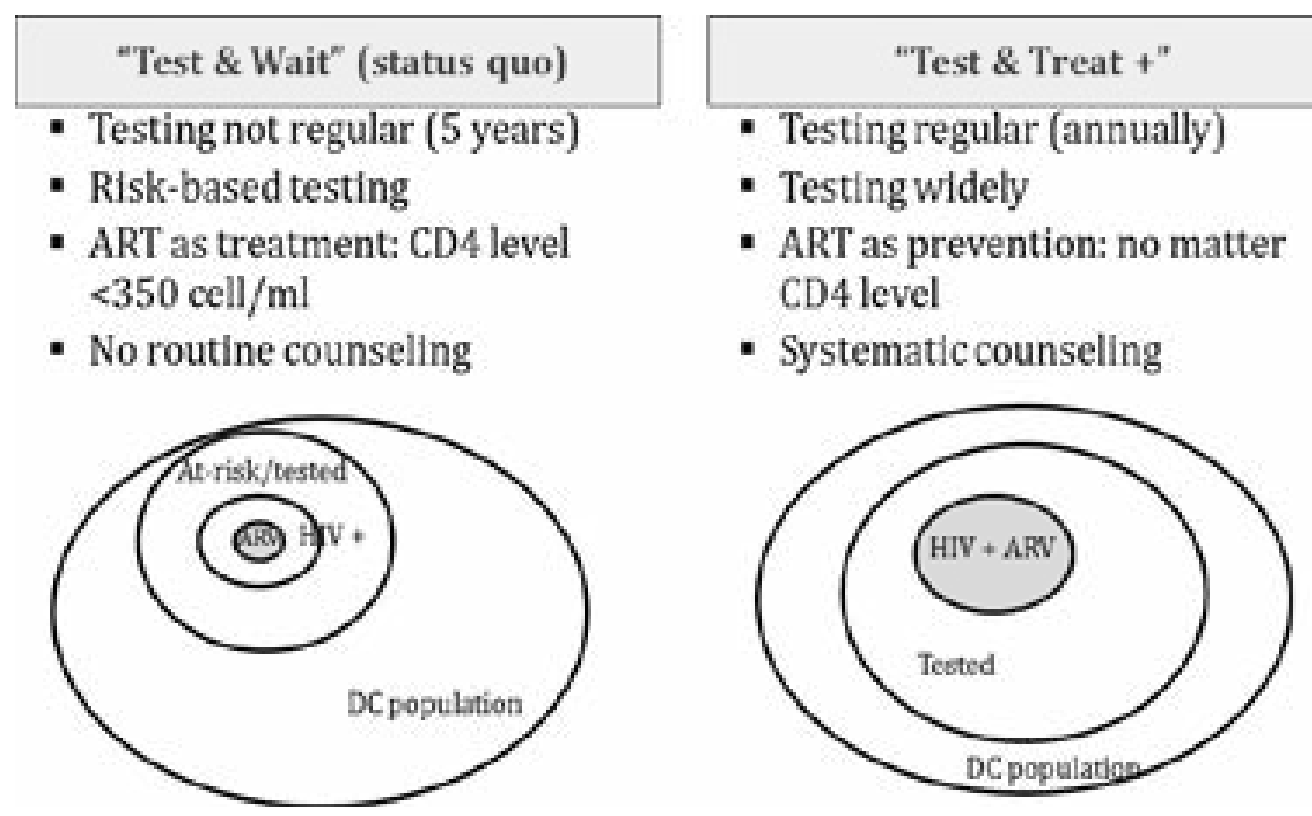
level between 350 and 500 cell $/ \mathrm{mm}^{3}$ (CDC 2006). Conversely, T\&T essentially consists of starting ARV earlier in the course of the disease with the objective of improving care and reducing transmission. It is notable that in 2013, the CDC changed the wording of its recommendations to take into account the latest epidemiological findings about the benefits of starting treatment early, including with a CD4 level above 500 cell $/ \mathrm{mm}^{3}$, stating that ARV "is recommended for all HIVinfected individuals to reduce the risk of disease progression" (CDC 2013a, E-4). A second distinct feature of T\&T is testing the general population rather than solely targeting high-risk populations. Third, the emphasis is put on a direct transition from the test site to a care provider where ARV is offered upon eligibility. Fourth, T\&T is also unique insofar as it includes a strong education component during screening and later at regular intervals during treatment, undertaken to ensure adherence to ARV.

T\&T programs are spreading around the world, particularly in countries with a high prevalence rate, such as South Africa, which is the first country with a nationwide T\&T initiative. In the US, the implementation of T\&T is still in its infancy, with pilot interventions in San Francisco and an ongoing three-year impact study (entitled TLC+) being undertaken by the NIH in the Bronx, NY; New York City; and Washington, DC (DAIDS 2010).

The high prevalence and incidence rates of HIV/AIDS in Washington, DC warrant continued policy efforts. While the preliminary findings from T\&T evaluations in the US and evidence from South Africa show the benefits of such a comprehensive approach, these benefits have not been monetized. Furthermore, researchers have highlighted the implementation challenges and the large financial implications of widespread T\&T. However, intervention and opportunity costs have yet to be analyzed systematically. Meanwhile, there are considerable budget constraints in Washington, DC; thus, finding an efficient prevention and care strategy is essential. Moreover, arrays of other innovative programs that fight the HIV/AIDS epidemic are also candidates for government funding. Consequently, undertaking an ex ante cost-benefit analysis of T\&T in Washington, DC is consistent with the evidence-based policy pledge of the current administration.

\section{Methods and Assumptions}

\section{Perspectives}

Given the nature of the intervention and the number of positive externalities linked to HIV/AIDS prevention and treatment, this ex ante cost-benefit analysis is taking a broad societal perspective. The most direct benefits are expected to accrue to individuals living with HIV/ AIDS, specifically those who are not yet aware of their status, as well as individuals who are at high risk but are not yet infected. Additionally, an intervention that limits the HIV/AIDS epidemic has a potential positive effect on the entire Washington, DC population, as well as on neighboring communities in Maryland and Virginia. Although benefits can spillover outside the metropolitan area, we decided to restrict the analysis to localized effects. With regards to costs, we can expect that not unlike other health programs in the district, they will be shared between $\mathrm{DOH}$, care providers, private insurers, Medicaid, and the individuals themselves. An Ex Ante Cost-Benefit Analysis

In assessing the costs and benefits of T\&T in Washington, DC, we face a number of methodological challenges. First, T\&T refers to a set of factors, rather than one well-defined program with a finite set of activities and a precise logic of intervention. Moreover, T\&T has thus far only been pilot-tested in a small group of cities in the US, and its expected impacts are still objects of evaluation. Therefore, this ex ante cost-benefit analysis relies on limited information, some of which stems 
from simulation studies whose conditions are likely to be different from real-world implementation. Nonetheless, the strong Monitoring and Evaluation component in the TLC+ impact study ongoing in Washington, DC is yielding promising preliminary results that were presented during the international conference on HIV/AIDS, held in Washington, DC in 2012. We use these findings as a basis for our analysis (Walensky et al. 2010; Kessler et al. 2012; DAIDS 2010; Greene et al. 2012). Additionally, this ex ante cost-benefit analysis taps into abundant epidemiological evidence of the preventive effectiveness of ARV regimens (Attia et al. 2009; Montaner et al. 2010; Granich et al. 2009). While the benefits of T\&T have yet to be monetized, a number of cost-effectiveness studies of interventions similar to T\&T have been undertaken (Walensky et al. 2007; Bendavid et al. 2010; Holtgrave et al. 1992). These studies offer a solid basis to appraise the costs of testing, treatment, and prevention interventions. Finally, the CDC and DOH have released a number of reports with up-to-date estimates of DC residents living with HIV/AIDS, engaged in care, and lost to care. These reports are key to this analysis (DOH 2012; HAHSTA 2012; The White House 2010).

\section{Theory of change}

While T\&T is designed to provide a comprehensive approach to prevention and treatment of HIV/AIDS, it is useful for the purposes of this analysis to decompose the intervention into a series of components, and to reconstruct the theory of change of the intervention. A model of the theory of change underlying T\&T is presented in Figure 2. A number of assumptions underlie this theory of change. First, the causal pathways from inputs and activities to shorter- and longer-term outcomes are linear but conditional upon a number of circumstances. Second, there is no unanticipated effect in terms of distorting behavior of being tested. In other words, we assume that people who have been tested are not more likely to engage in risky behavior than people who have not been tested. This is a plausible assumption given that testing is embedded in a broader counseling session.

Figure 2: Test \& Treat Simplified Theory of Change

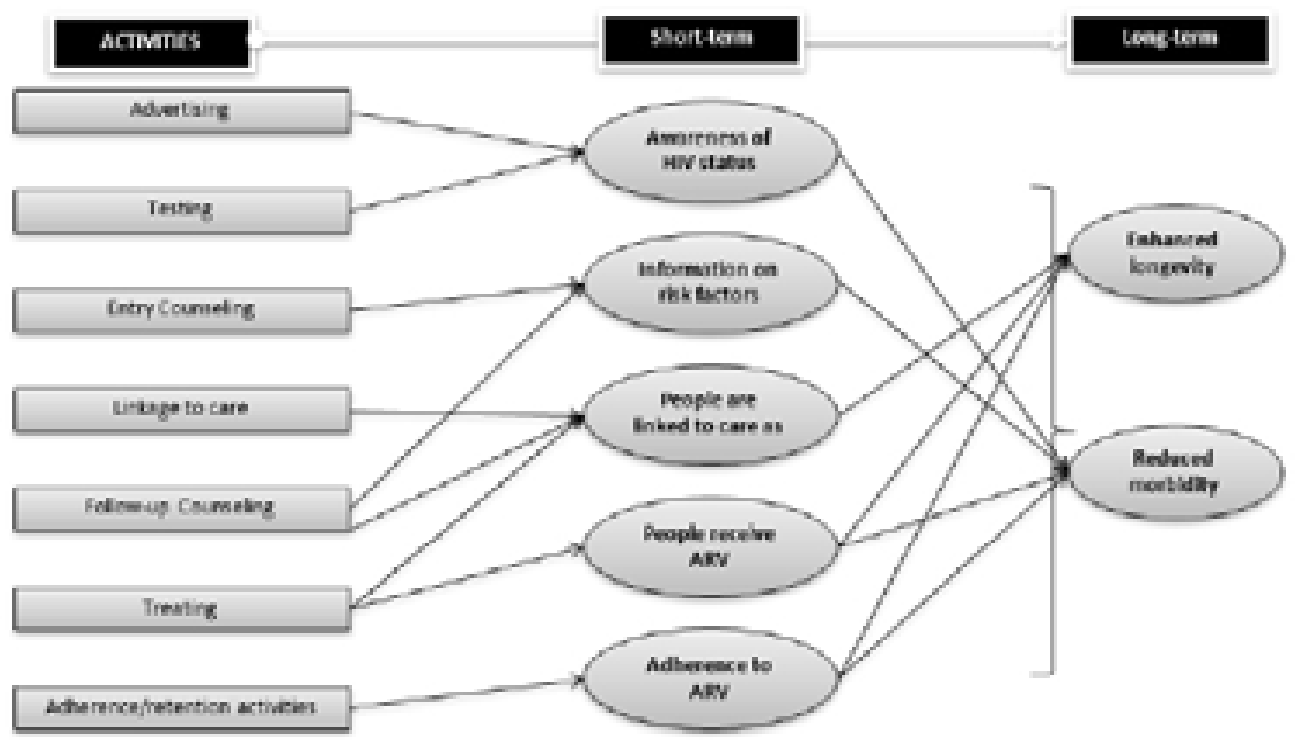


Third, ARV regimens improve well-being, provided that health improvement linked to suppression of viral load is higher than pain from side effects. Finally, ARV regimens increase longevity, provided non-adherence does not lead to development of resilient breeds of the virus.

\section{Analyzing future benefits and costs}

The ex ante cost-benefit analysis presented here primarily includes costs that are spread over a number of years and benefits that occur at an unknown future date. In the main part of the analysis, we assume that costs and benefits would be largely concomitant, and thus no discounting is introduced. In the sensitivity analysis, we introduce discounting in order to compare the dollar value of costs and benefits received in different time periods to their present values. Finally, while all costs in this analysis are subject to inflation over time, progress by pharmaceutical companies and the development of generic ARV regimens have led to significant price reductions in the past decade that are likely to continue. In this analysis, all dollar amounts are expressed in 2010 dollars and the discounting rates introduced in the sensitivity analysis are real interest rates (rates of 3 percent and 5 percent are tested).

\section{Results}

\section{Program Costs}

The most significant cost associated with the implementation of T\&T is undeniably the lifetime cost of treatment, driven mostly by the cost of ARV. This cost is measured with the widely used estimate of $\$ 379,668$ presented by the CDC as the baseline (CDC 2013b). Given that T\&T operates under the premise of automatic treatment upon diagnosis, estimating the cost of treatment involves an assessment of the increased length of medication per individual compared to the CDC baseline. As it is impossible to measure precisely this duration without access to the actual records of the diagnosed and their CD4 level at diagnosis, we estimate an average increase in the duration of treatment of 15 months. This estimate is plausible given the recent progress in early diagnosis, which allows the identification of infected patients before they reach the critical level of 350 cell $/ \mathrm{mm}^{3}$. With regards to the price of medicines, the NIH routinely publishes a comprehensive price list for major ARV regimens in the US, including generics (NIH 2013). The costs of treatment range from $\$ 360$ to $\$ 2,840$ per month, depending on the efficacy and brand of the regimen. We use a value of $\$ 1,500$ as a price average for the base case analysis, which puts the lifetime cost of treatment under T\&T at $\$ 402,168$.

Other direct program costs include those associated with testing, linkage to care, and retention in care. These variable costs are measured using the CDC guidelines for cost-effectiveness analysis of HIV/AIDS, which contain recommended cost values for screening, testing, linkage to care, and retention activities, as laid out in Table 1 (CDC 2013b). These costs include staff and material costs and sum up to $\$ 12,400$ per diagnosed patient.

Such types of prevention and treatment interventions can also generate indirect costs in the form of negative externalities or side effects. For instance, previous studies have mentioned the risk that non-adherence to antiretroviral medicines can result in the development of breeds of the virus that are resistant to any type of treatment. Additionally, ARVs are notorious for their strong side effects, which can trigger additional medical costs and possibly foregone earnings. Nonetheless, neither of these indirect costs are integrated into this analysis because they are particularly difficult to assess and measure quantitatively. Moreover, newer generations of ARV have much fewer and weaker side effects, which we assume are negligible in com- 
Table 1: Stakeholders Summary for Costs and Benefits

\begin{tabular}{|c|c|}
\hline Costs & Paid By \\
\hline Direct Program Costs & \multirow{7}{*}{$\begin{array}{l}\text { Shared between DOH, care providers, } \\
\text { private insurance, Medicaid, patients }\end{array}$} \\
\hline Screening & \\
\hline Analysis of test results & \\
\hline Linkage to care & \\
\hline Retention/Adherence activities & \\
\hline Lifetime ARV & \\
\hline Indirect Program Costs & \\
\hline $\begin{array}{l}\text { Negative externality of non-adherence (de- } \\
\text { velopment of resistant breed of the virus) }\end{array}$ & DC population at large \\
\hline Negative side effects of ARV & \multirow{3}{*}{ Individuals diagnosed with HIV/AIDS } \\
\hline Stigma & \\
\hline Psychological costs of disease & \\
\hline Benefits & Accrued By \\
\hline Increase in Longevity & \multirow[b]{2}{*}{ Individuals diagnosed with HIV/AIDS } \\
\hline $\begin{array}{l}\text { Increase in number of Quality Adjusted } \\
\text { Life-Year }\end{array}$ & \\
\hline Reduced Incidence of HIV/AIDS & \multirow{3}{*}{ DC population at large } \\
\hline Avoided cost of lifetime treatment & \\
\hline Avoided loss in Quality Adjusted Life-Year & \\
\hline
\end{tabular}

parison to the symptoms of opportunistic diseases associated with HIV/AIDS.

The next stage of cost estimation consists of determining the number of people affected by each level of T\&T. The initial step of T\&T consists of a mass screening for HIV/AIDS, which takes the form of a cheek swab test, during which a short educative module is also implemented. The HIV/AIDS, Hepatitis, STD and TB Administration (HAHSTA) 2011 annual report estimated the number of publicly funded tests for HIV/AIDS at 122,000 (HAHSTA 2012). Given that two key idiosyncrasies of T\&T are to extend screenings from the "at-risk population" to the population at large and to test more regularly, we assume a 30 percent increase in the number of tests, which is consistent with the numbers reported by Kessler et al. for the TLC+ pilot imple- mented in the Bronx, New York (Kessler et al. 2012).

For the subsequent phases of T\&T, we use the "Treatment Cascade" developed by Gardner et al. (2001). This framework provides a model of attrition at every stage of the care spectrum, from screening for HIV/AIDS to complete viral suppression. While Gardner et al. apply this framework at the national level, the HAHSTA annual report provides specific probabilities of retention at each step that are specific to Washington, DC and are used as our measure of retention under the status quo. For this analysis, we provide three retention scenarios: a "base" scenario, which relies on the preliminary data provided by the TLC+ pilot evaluation in Washington, DC (Walensky et al. 2010; Kessler et al. 2012; Greene et al. 2012), as well as a "pessimistic" and a 
"best-case" scenario. While both of these are presented in the sensitivity analysis, Figure 3 displays the probabilities used in the base scenario.

HAHSTA reports that for 2012, 83 percent of 842 people diagnosed with HIV/AIDS in DC were linked to care within one year of diagnosis. However, 75 percent of them were ultimately lost in care. Out of those retained, 92 percent are eligible for ARV treatment, and 145 (90 percent) will ultimately be viral-suppressed. In the base scenario, we assume that widespread screening for HIV/AIDS will tend to increase the number of positive diagnoses by 15 percent to reach 960 cases. Given that 30 percent of the population living with HIV/AIDS in the district is unaware of their status, 15 percent is a plausible assumption. Secondly, the main difference with the status quo is that 100 percent of people who are retained in care are eligible for ARV-in view of the automatic linkage to treatment upon diagnosis, which underpins T\&T. Ultimately, 397 people will be virally suppressed. This represents a 41 percent performance rate, which is in line with Walensky et al.'s medium scenario (2010).

\section{Benefits}

As described above, there are two major benefits to T\&T from a societal standpoint: increased longevity for individuals living with HIV/AIDS and reduced incidence of the virus within Washington, DC. We estimate the expected increase in longevity for people who are already infected with HIV/AIDS as the number of life-years gained attributable to T\&T. To obtain this measure, we rely on the rigorous impact evaluation of the TLC+ program taking place in Washington, DC. The design of this study is a randomized controlled trial, which enables causal inference. Using these data and a mathematical simulation model, Walensky et al. found that T\&T can increase longevity between 1.1 and 5.8 years, depending on the effectiveness of the ARV selected and the adherence of the patient (2010). For the base scenario, we employ an estimate of 2.7 life-years gained compared to the status quo. We

Figure 3: Treatment Cascade of the Test \& Treat Strategy (Base Scenario)

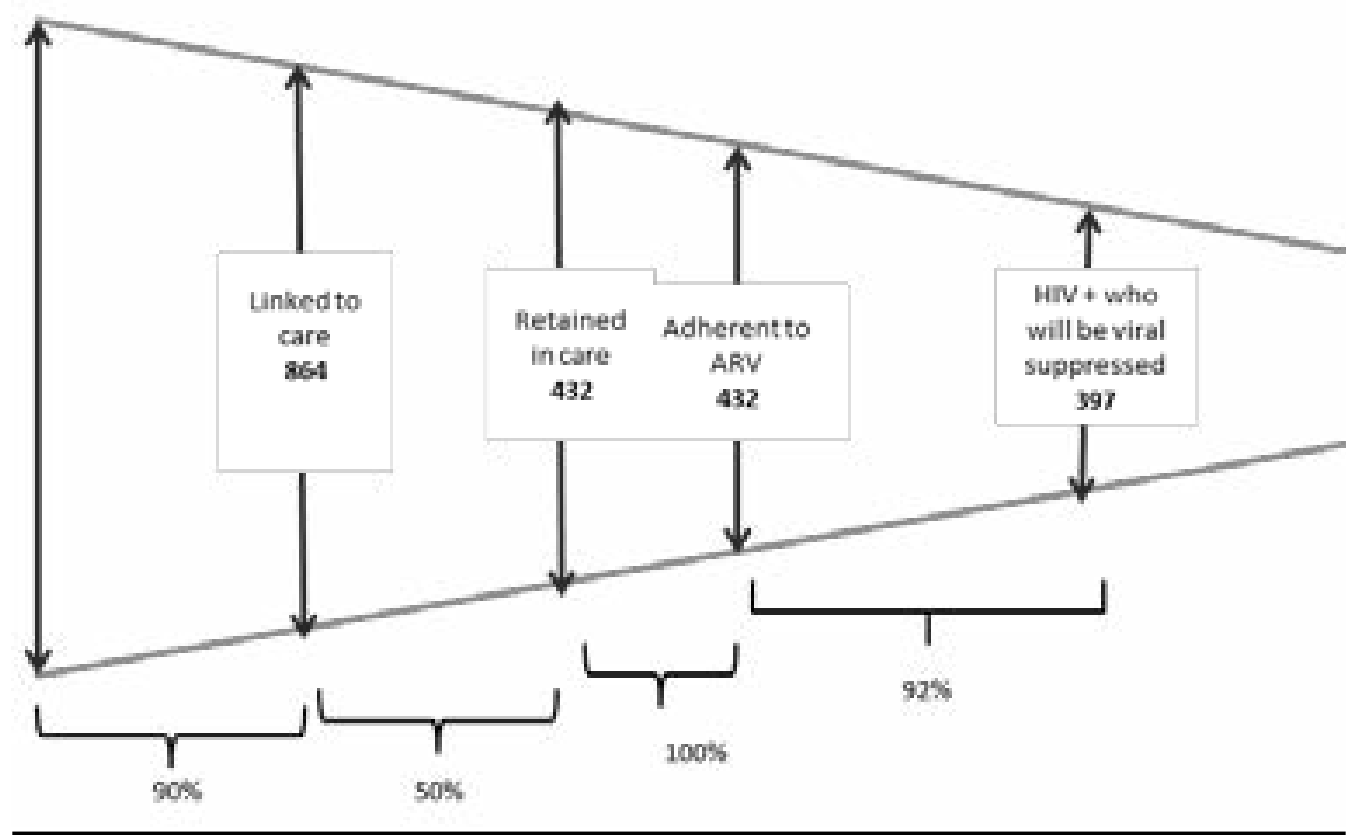


assume that only patients who are fully retained in care and adherent to ARV fully benefit from this increase in longevity. Consequently, our estimate of the longevity benefit is quite conservative because it ignores life-years gained by patients who partially adhere to the regimen.

In the HIV/AIDS literature, and in accordance with the CDC guidelines, the quality of an added year of life is assessed by Quality Adjusted Life Year (QALY). This method consists of weighing the number of years gained according to the quality attributed to them by individuals. The assumption underlying this principle is that people are willing to pay less for an additional year of life in illness than a year of perfect health. The quality index ranges from o to 1 . We rely on the results of a contingent valuation survey, which estimated that, on average, people living with HIV/AIDS value one additional life-year at 0.82 (Tengs 2002). This survey was conducted in the early 2000s, and we expect that it is a conservative measure given the progress of ARV regimens and their effectiveness in keeping people living with HIV/AIDS healthier with limited side effects. The weighted QALY adopted in the base case is thus 2.214.

We next monetize this value by using the Value of Statistical Life-Year (VSLY). This measure is somewhat controversial because it attributes a higher value to the life of a young person than to the life of an older one. Nonetheless, it is widely used and the Office of Management and Budget (OMB) encourages agencies to provide estimates of both VSLY and an overall measure of the Value of Statistical Life (VSL) in cost-benefit analyses (OMB 2003). The base case adopts the VSLY proposed by Boardman et al. as the best point estimate for policy purposes in the US, which amounts to $\$ 234,136$ per year per person. It is based on a VSL of $\$ 5$ million with a discount rate of 3.5 percent and a 40-year life expectancy (2011).
The Treatment Cascade described above and described in Figure 3 provides an estimate of the number of patients who will ultimately become virally suppressed each year. Compared to the status quo, 252 additional individuals become virally suppressed under this estimate. The total non-discounted benefit from $T \& T$ in terms of increased longevity amounts to $\$ 130,631,030$.

The second benefit of this intervention lies in the reduced incidence of the virus expected from the use of treatment as prevention. This benefit can also be conceptualized as the gain from the number of infections averted. There are two types of gains associated with a reduction in the number of infections: treatment costs averted and the value of the years that would otherwise have been lost due to HIV/AIDS. The formula below sums up this measurement strategy: Number of infections averted $x$ (lifetime treatment cost + value of QALYs that would have been lost).

The CDC estimate of $\$ 379,668$ serves as our measure of lifetime treatment cost in this counterfactual scenario (CDC 2013b). With regards to the number of years that would otherwise have been lost to the disease, we compare the life expectancy at diagnosis under the status quo, as described in Walensky et al. with the average life expectancy in the US (Walensky et al. 2010; CDC 2012a). On average, individuals with HIV/AIDS live 13.34 fewer years than those not infected. Adjusting this value for quality of life, the estimate is 10.9 QALYs that are gained by avoided infection. The overall gain per infection averted consequently amounts to $\$ 2,940,834$. This value will be revisited using more conservative and generous estimates of VSLY in the sensitivity analysis.

In the remaining step, we estimate the number of infections averted per year that can be attributed to T\&T. This computation is difficult due to the scarcity of reliable information on the incidence of HIV/AIDS in Washington, DC, which 
is required to establish a baseline. We use the CDC's approximation of its incidence rate of HIV/AIDS, which was computed based on the number of annual diagnoses for the past decade, as well as an estimate of average time-lapse between diagnosis and probable infection. Based on this estimate, which is likely to be an underestimation, Washington, DC has one of the highest incidence rates of HIV/AIDS in the country, at 155.6 infections per 100,000 inhabitants (CDC 2012b). Applying this incidence rate to Washington, DC's population of 632,323 , we estimate the number of new infections per year to be 984 (U.S. Census Bureau 2012).

The number of infections avoided depends on the efficacy of T\&T in two key areas: identifying and treating people who are already infected but are unaware of their status, and raising awareness among the population at risk of infection through education and screening. The interaction between the multiple parameters that interplay in transmission patterns makes it particularly complex to estimate the number of infections averted ex ante. Consequently, we rely on secondary data for our estimation. In the T\&T experiment carried out in the Bronx, NY, Kessler et al. project that the strategy could reduce the number of infections by up to 23 percent over 20 years in their base case; in their best-case scenario, this estimate is up to 81 percent (2012). In their simulation for Washington, DC, Walensky et al. found that within five years, the community viral load for the district could be curbed by 48.8 percent in their base scenario and up to 65 percent in a more optimistic scenario (2010). While this measure cannot be readily translated to infections averted, it confirms the high efficacy of the strategy as a prevention mechanism. We opt for a value of 5 percent annual decrease in infections as a plausible estimate for the start of the program. The number of infections would decrease at an increasing rate over time as the community viral load begins shrinking, but this would take a num- ber of years, and we thus opt for a constant rate. Overall, 50 infections averted per year represents an undiscounted benefit of $\$ 147,041,744$. The combined benefits of increased longevity and reduced incidence amount to $\$ 277,672,774$. Given the costs and benefits detailed so far, the vaccination program produces positive net benefits of $\$ 148,218,398$. Table 2 summarizes these costs and benefits.

\section{Sensitivity analysis}

The spread of HIV/AIDS is an extremely dynamic phenomenon, which renders causal processes at play in prevention and treatment interventions non-linear. Consequently, any attempt to estimate the costs and benefits of such an intervention is particularly sensitive to even the smallest parameter change, which warrants a robust sensitivity analysis. We organize the sensitivity analysis in two parts. In the first part, the base scenario is compared to two additional scenarios: a pessimistic scenario and a best-case scenario. In the second part, we submit each scenario to a discounting procedure using both the 3 percent discount rate recommended by OMB and a more conservative discount rate of 5 percent.

For the best-case scenario, we adopt a generous estimate of individuals reached and retained into care. While this measure tends to increase benefits substantially, it also raises costs, as most benefits largely depend on the number of individuals reached without possibilities of economies of scale. In this scenario, we retain the same VSLY as in the base case, but we increase the number of life-years gained from 2.7 to 5.3. With regards to costs, the best-case scenario applies a lifetime treatment cost of $\$ 390,000$ and a retention activities cost of $\$ 3,000$. Conversely, in the pessimistic scenario, we significantly reduce the retention rates at each level of the Treatment Cascade, which results in diminished benefits and costs. We use a conservative value of VSLY at \$95,640, which is consistent 
Table 2: Summary of Costs and Benefits

\begin{tabular}{|c|c|c|c|}
\hline \multicolumn{4}{|l|}{ Costs } \\
\hline & Cost/unit & \# of units & $\begin{array}{c}\text { Total compared to } \\
\text { status quo }\end{array}$ \\
\hline Screening & 150 & 120,542 & $\$ 8,806,500$ \\
\hline Positive diagnosis & 5,000 & 960 & $\$ 625,000$ \\
\hline Linkage to care & 1,250 & 864 & $\$ 731,000$ \\
\hline Retention/adherence activity & 6,000 & 432 & $\$ 2,505,500$ \\
\hline Lifetime treatment $(\mathrm{r}=\mathrm{O} \%)$ & 402,168 & 432 & $\$ 116,786,376$ \\
\hline Total annual costs & & & $\$ 129,454,376$ \\
\hline \multicolumn{4}{|l|}{ Benefits } \\
\hline \multicolumn{4}{|l|}{ Increased Longevity } \\
\hline Life-year gained & & & 2.7 \\
\hline QALY & & & 0.82 \\
\hline VSLY & & & $\$ 234,136$ \\
\hline * of persons with viral suppression & & & 252 \\
\hline Benefit in Longevity & & & $\$ 130,631,030$ \\
\hline \multicolumn{4}{|l|}{ Decreased Morbidity } \\
\hline Lifetime treatment cost per HIV case & & & 379,668 \\
\hline QALY of lost years per infection & & & 0.82 \\
\hline Number of lost years & & & 13.34 \\
\hline VSLY & & & $\$ 234,136$ \\
\hline Number of infections averted & & & 50 \\
\hline Benefit in Morbidity & & & $\$ 147,041,744$ \\
\hline Total annual benefits & & & $\$ 277,672,774$ \\
\hline Net Benefits & & & $\$ 148,218,398$ \\
\hline
\end{tabular}

with the lower bound of values used in cost-effectiveness analysis (Mrozek \& Taylor 2002; Miller 2000). In addition, we incorporate a gain in life-years of 1.1, which is consistent with Walensky et al.'s most conservative estimate (2010). Using these assumptions, the cost of treatment equals $\$ 500,000$, and retention activities amount to $\$ 10,000$. These parameters are summarized in Table 3 on the following page.

As demonstrated in Table 3, T\&T is cost-efficient under two of the three scenarios. In the best-case scenario, the cost-benefit ratio reaches 3.45 with a positive net benefit of $\$ 511,063,945$. However, under the pessimistic scenario, benefits do not cover the costs of the program and incur a negative net benefit of $\$ 30,686,657$ with a cost-benefit ratio of 0.53 . Given the relatively large number of parameters and probabilities included in these computations, a break-even analysis was not deemed relevant and is not reported here.

\section{Discounting}

In the main part of the analysis, costs and benefits were assumed to be largely simultaneous, and thus no discounting was introduced. In this section of the sensitivity analysis, both costs and benefits are discounted using two 
Table 3: Partial Sensitivity Analysis Parameters

\begin{tabular}{|l|c|c|c|}
\hline & \multicolumn{3}{|c|}{ Scenarios } \\
\hline Benefits & Base & Pessimistic & Best \\
\hline VSLY $(\$)$ & 234,136 & 95,640 & 234,136 \\
\hline Life-year gained & 2.7 & 1.1 & 5.3 \\
\hline Infections averted & 50 & 20 & 70 \\
\hline Virally suppressed & 265 & 75 & 505 \\
\hline \multicolumn{1}{|c|}{ Linked } & $90 \%$ & $85 \%$ & $95 \%$ \\
\hline \multicolumn{1}{|c|}{ Retained } & $50 \%$ & $30 \%$ & $75 \%$ \\
\hline \multicolumn{1}{|c|}{ Virally suppressed } & $92 \%$ & $90 \%$ & $95 \%$ \\
\hline Costs & 402,168 & 500,000 & 390,000 \\
\hline Lifetime treatment & 6,000 & 10,000 & 3,000 \\
\hline Retention activities & \multicolumn{3}{|c|}{} \\
\hline
\end{tabular}

real discount rates: 3 percent, as recommended by OMB (2003), and a more conservative 5 percent rate. While most program costs are incurred in the first year of the intervention, the cost of ARV is spread over the entire duration of the treatment. The time between diagnosis and expected death under T\&T is estimated to be 29 years. Consequently, we divide the lifetime treatment cost by this number of years and discount over the entire duration of treatment. As for benefits, the overall dollar amount was divided over the number of QALYs gained under each scenario and discounted over the respective period. For instance, in the best-case scenario, patients who are viral suppressed can expect to live five years longer than under the status quo, which is valued at $\$ 234,136$ annually. When discounting over five years at a rate of 3 percent, the total discounted benefit amounts to $\$ 527,488$, compared to the $\$ 1,170,680$ undiscounted value.

Table 4 presents the results of the sensitivity analysis including the two discounting procedures. While discounting leads to lower net benefits and cost-benefit ratios in the base and bestcase scenarios, it does not fundamentally change the results. Even with a conserva- tive 5 percent discount rate, T\&T remains efficient in the base scenario.

\section{Limitations}

The limitations in this analysis stem from the omission of data that are relevant but difficult, if not impossible, to quantify and monetize. For instance, there is abundant evidence from qualitative health research that individuals being tested and diagnosed for HIV/AIDS incur significant psychological and social costs, which should not be underestimated (Ransom et al. 2005; Siegel et al. 1998). In particular, individuals living with HIV/ AIDS must bear the cost of stigma and its consequences on employment, housing, and access to health insurance. Other psychological costs stem from being dependent on medicines and being at the mercy of side effects that can have deleterious effects on body and self-images, and such costs should in principle not be overlooked (De Wit and Adam 2008; Wilson \& Hutchinson 2010). Presumably, T\&T strategies that render treatment automatic and systematize testing can compound these effects. On the other hand, this analysis did not take into account the possible herding effect that could develop over time as fewer people become infected with HIV/AIDS. 
Table 4: Partial Results of Sensitivity Analysis: Net Benefits and Cost-Benefit Ratios

\begin{tabular}{|c|c|c|c|c|c|c|}
\hline \multicolumn{9}{|c|}{ Scenario } \\
\hline $\begin{array}{c}\text { Discount } \\
\text { Rate }\end{array}$ & \multicolumn{2}{|c|}{ Base } & \multicolumn{2}{c|}{ Pessimistic } & \multicolumn{2}{c|}{ Best } \\
\hline & Net Benefits & Ratio & Net Benefits & Ratio & Net Benefits & Ratio \\
\hline $\mathrm{r}=0 \%$ & $\$ 148,218,398$ & 2.14 & $(\$ 30,686,657)$ & 0.53 & $\$ 511,063,945$ & 3.45 \\
\hline \multirow{2}{*}{$\mathrm{r}=3 \%$} & $\$ 69,376,594$ & 1.73 & $(\$ 31,646,522)$ & 0.4 & $\$ 234,456,247$ & 2.59 \\
\cline { 2 - 8 } & $\$ 73,611,965$ & & & & & \\
\hline \multirow{2}{*}{$\mathrm{r}=5 \%$} & $\$ 32,321,353$ & 1.42 & $(\$ 27,176,763)$ & 0.38 & $\$ 123,860,050$ & 2.05 \\
\cline { 2 - 8 } & $\$ 34,891,486$ & & & & & \\
\hline
\end{tabular}

A more focused perspective may have yielded a somewhat different set of findings. For instance, providing a disaggregated account of costs and benefits by race and gender would have allowed us to identify beneficiaries and potential spillover effects more clearly. Indeed, it is well-established that Black residents in DC are most heavily impacted by the disease, as well as the most at-risk. In 2010, they represented 75 percent of those living with HIV/AIDS while only making up 46 percent of the city's population (HAHSTA 2012). Moreover, although the incidence of HIV/AIDS is greater among men in general, prevalence is higher among Black women (2.6 percent) compared with white men (2.4 percent). Racial disparities should be analyzed in future research.

\section{Policy Recommendations}

This ex ante cost-benefit analysis concludes that under a plausible set of circumstances, it is economically efficient to implement T\&T in Washington, DC. Benefits outweigh costs with a value of statistical life year that is widely endorsed in the literature. Moreover, we opted for relatively conservative estimates of the number of infections averted and the number of individuals who live with HIV/AIDS and achieve viral suppression. Therefore, taking solely into account the efficiency criteria, it is reasonable to recommend the scale- up of this strategy to the district as a whole.

However, several other policy goals should be taken into account when deciding to change policy guidelines, which directly affect the lives of one in 20 DC residents and have consequences for the entire city. First, any decisions to scale up should await the results of the TLC+ impact evaluation with rigorous analysis of effects and an empirically grounded assessment of the scale of these effects, as opposed to simple simulation results. Second, given that the success of such an intervention largely relies on the adherence of patients to ARV, particular attention should be given to retention activities. This implies enhancing the information base on the obstacles that individuals living with HIV/AIDS face in terms of access to, and observance of, their medical prescriptions. Qualitative research on stigma and the psychological costs of treatment should also be undertaken.

Finally, the feasibility of such an intervention depends on the coordination of efforts within the Washington, DC Metropolitan Area under the Ryan White Plan. This area includes northern and western Virginia, as well as five border counties in Maryland. While the incidence and prevalence rates of HIV/AIDS are also high in the district's periphery, they are significantly lower than in DC. T\&T's efficiency may fare worse in com- 
munities with lower rates.

This analysis alone does not provide a sufficient basis for making a policy decision to implement a complex and dynamic T\&T intervention. It should, however, be considered as a preliminary foundation upon which further analyses may build.

\section{References}

Attia, Suzanna, Matthias Egger, Monika Müller, Marcel Zwahlen, and Nicola Low. 2009. "Sexual Transmission of HIV According to Viral Load and Antiretroviral Therapy: Systemic Review and Meta-Analysis.” AIDS 23(11):1397-404.

Boardman, Anthony, David Greenberg, Aidan Vining, and David Weimer. 2011. CostBenefit Analysis, Concepts and Practice, 4th edition. Upper Saddle River, New Jersey: Prentice Hall.

Bendavid, Erin, Margaret L. Brandeau, Robin Wood, Douglas K. Owens. 2010. "Comparative Effectiveness of HIV Testing and Treatment in Highly Endemic Regions." Arch Intern Med 170(15):1347-1354.

Centers for Disease Control and Prevention (CDC). 2006. "Revised Recommendations for HIV Testing of Adults, Adolescents, and Pregnant Women in Health-Care Settings.” Last modified September 22, 2006. http://www.cdc.gov/mmwr/ preview/mmwrhtml/rr5514a1.htm.

. 2012a. "Health, United States, 2012: With Special Feature on Emergency Care." http://www.cdc.gov/nchs/data/hus/hus12.pdf\#018.

. 2012b. "HIV Incidence 2007-2010 (through 2010)." Last modified December 3, 2013. http://www.cdc.gov/hiv/library/slideSets/index.html.

. 2012c. "HIV Surveillance Report: Diagnoses of HIV Infection and AIDS in the United States and Dependent Areas, 2010, Vol. 22.” Washington, DC: CDC. . 2013a. "Guidelines for the Use of Antiretroviral Agents in HIV-1-Infected Adults and Adolescents." Last modified February 12, 2013. http://aidsinfo.nih.gov/ guidelines/html/1/adult-and-adolescent-arv-guidelines/o.

. 2013b. "HIV Cost-effectiveness.” Last modified April 16, 2013. http://www.cdc. gov/hiv/topics/preventionprograms/ce/index.htm\#Overview.

De Wit, J.B.F., and P.C.G. Adam. 2008. "To Test or Not to Test: Psychosocial Barriers to HIV Testing in High-Income Countries." HIV Medicine 9(2):20-29.

District of Columbia Department of Health (DOH). 2012. "Washington DC Regional Eligible Metropolitan Area. 2012-2014 Comprehensive HIV Care Plan.” Washington, DC: Department of Health.

Division of AIDS (DAIDS), U.S. National Institute of Allergy and Infectious Diseases (NIAID), U.S. National Institutes of Health (NIH). 2010. "HPTN 065, TLCPlus: A Study to Evaluate the Feasibility of an Enhanced Test, Link to Care, Plus Treat Approach for HIV Prevention in the United States: A Study of the HIV Prevention Trials Network.” Washington, DC: NIH.

Gardner, Edward M., Margaret P. McLees, John F. Steiner,Carlos Del Rio, and William J. Burman. 2011. "The Spectrum of Engagement in HIV Care and its Relevance to Test-and-Treat Strategies for Prevention of HIV Infection.” Clinical Infectious Diseases 52:793-800.

Granich, R .M., G. F. Gilks, C. Dye, and K. M. DeCock. 2009. "Universal Voluntary HIV Testing with Immediate Antiretroviral Therapy as a Strategy for Eliminating HIV Transmission: A Mathematical Model.” The Lancet 373(9657):48-57. 
Greene, E., K. Buchacz, T. Gamble, G. Breauchamp, L. McKinstry, A. Wood, E. Telzak, B. Branson, and W. El-Sadr. 2012. "Linkage-to-Care and ART Adherence Practices at Participating Sides in the HPTN 065 (TLC-Plus) Study." Presented at the 19th International AIDS Conference.

HIV/AIDS, Hepatitis, STD, and TB Administration (HAHSTA), District of Columbia Department of Health. 2012. “Annual Report 2011.” Washington, DC: Department of Health.

Holtgrave, David R., Ronald O. Valdiserri, A. Russell Gerber, and Alan R. Hinman. 1993. "Human Immunodeficiency Virus Counseling, Testing, Referral, and Partner Notification Services: A Cost-Benefit Analysis." Arch Intern Med 153:1225-1230.

Health Resources and Services Administration, U.S. Department of Health and Human Services. 2012. "Test and Treat: A New Paradigm for Slowing the Spread of HIV.” Accessed January 23, 2013. http://hab.hrsa.gov/newspublications/careactionnewsletter/hab_test_and_treat_january_careaction_pdf.pdf.

Kaiser Family Foundation. 2012. "The HIV/AID Epidemic in Washington, DC" Last modified July 2012. http://kaiserfamilyfoundation.files.wordpress. com/2013/o1/8335.pdf.

Kessler, Jason, Julie E. Myers, Kimberly A. Nucifora, Nana Mensah, Alexis Kowalski, Monica Sweeney, Christopher Toohey, Amin Khademi, Colin Shepard, Blayne Cutler, and R. Scott Braithwaite. 2012. "Expanded Antiretroviral Therapy in New York City: Modeling the Impact.” Presentation at the Conference on Retroviruses and Opportunistic Infections.

Lange, Joep M.A. 2011. “'Test and Treat': Is It Enough?” Clinical Infectious Diseases 52(6):801-802.

Miller, Ted R. 2000. "Variations Between Countries in Values of Statistical Life.” Journal of Transport Economics and Policy 34(2) 2000:169-188.

Montaner, J. S., V.D. Lima, R. Barrios, B. Yip, E. Wood, T. Kerr, K. Shannon, P.R. Harrigan, R.S. Hogg, P. Daly, and P. Kendall. 2010. "Association of Highly Active Antiretroviral Therapy Coverage, Population Viral Load, and Yearly New HIV Diagnoses in British Columbia, Canada: A Population-Based Study." The Lancet 376(9740):532-539.

Mrozek, Janusz R., and Laura O. Taylor. 2002. "What Determines the Value of Life? A Meta-Analysis.” Journal of Policy Analysis and Management 21(2):253-270.

Mugavero, Michael J., Hui-Yi Lin, Jeroan J. Allison, James H. Willig, Pei-Wen Chang, Malcolm Marler, James L. Raper, Joseph E. Schumacher, Maria Pisu, and Michael S. Saag. 2007. "Failure to Establish HIV Care: Characterizing the "No Show’ Phenomenon.” Clinical Infectious Diseases 45:127-30.

HHS Panel on Antiretroviral Guidelines for Adults and Adolescents, Office of AIDS Research Advisory Council (OARAC), U.S. Department of Health and Human Services. 2013. "Guidelines for the Use of Antiretroviral Agents in HIV-1-Infected Adults and Adolescents."

Office of Management and Budget. 2003. "Circular A-4: Regulatory Analysis."

Piot, Peter, Michael Bartos, Heidi Larson, Debrework Zewdie, Purnima Mane. 2008. "Coming to Terms with Complexity: A Call to Action for HIV Prevention." The Lancet 9641(372):845-859.

Project Inform. 2010. “TLC+: Testing, Linkage to Care and Treatment.” Accessed January 27, 2013. http://www.projectinform.org/tlc+.

Ransom, Jane E., Bobbie Siler, Rosalind M. Peters, and Mary Jo Maurer. 2005. 
"Worry: Women's Experience of HIV Testing." Qualitative Health Research 15(3),382-93.

Schackman, Bruce R., Kelly A. Gebo, Rochelle P. Walensky, Elena Losina, Tammy Muccio, Paul E. Sax, Milton C. Weinstein, George R. Seage III, Richard D. Moore, and Kenneth A. Freedberg. 2006. "The Lifetime Cost of Current Human Immunodeficiency Virus Care in the United States." Medical Care 44(11):990-7.

Siegel, K., V.H. Raveis, and E. Gorey. 1998. "Barriers and Pathways to Testing Among HIV-Infected Women.” AIDS Education and Prevention 10(2),114-27.

Tengs, Tammy O., and Ting H. Lin. 2002. "IN1 Meta-Analysis of Utility Estimates for HIV/AIDS and Stroke." Value in Health 5(3):122.

United States Census Bureau. 2013. "State \& County QuickFacts: District of Columbia." Last modified June 27, 2013. http://quickfacts.census.gov/qfd/states/1100o. html.

Walensky, Rochelle P., Kenneth A. Freedberg, Milton C. Weinstein, A. David Paltiel. 2007. "Cost-Effectiveness of HIV Testing and Treatment in the United States." Clinical Infectious Diseases 45:S248-255.

Walensky, Rochelle P., A. David Paltiel, Elena Losina, Bethany L. Morris, Callie A. Scott, Erin R. Rhode, George R. Seage, and Kenneth A. Freedberg. 2010. “Test and Treat DC: Forecasting the Impact of a Comprehensive HIV Strategy in Washington DC.” Clinical Infectious Diseases 51(4):392-400.

The White House. 2010. "National HIV/AIDS Strategy for the United States."

Wilson, Holly Skodol, Sally A. Hutchinson, and William L. Holzemer. 2002. "Reconciling Incompatibilities: A Grounded Theory of HIV Medication Adherence and Symptom Management." Qualitative Health Research 12(10):1309-22.

Estelle R. Raimondo is a $\mathrm{PhD}$ candidate at the George Washington University's Trachtenberg School of Public Policy and Public Administration with a focus on program evaluation. Estelle also works as a graduate research assistant in the GWU Office of Survey Research and Analysis. Prior to joining GWU, she served as an associate evaluation specialist at the United Nations Educational, Scientific and Cultural Organization (UNESCO) where she conducted a number of evaluations notably in Africa. Estelle holds a dual Master's degree in international affairs from Sciences Po Paris and Columbia University (New York) that she earned as a Fulbright scholar.

The author extends her appreciation to the Policy Perspectives editorial team, particularly Tony Mastria, for bringing this project to fruition and for their iterative review of the article which tremendously enhanced its quality. The author expresses her gratitude to Professor Kathy Newcomer for taking the time out of her busy schedule to provide insightful comments on the paper. Finally, the author would like to thank her partner, Dominique, whose work on HIV prevention in Washington, DC, inspired the topic for this article, and Professor Joseph Cordes for his teaching of cost-benefit analytical skills in the course where this paper originated. 